Revista

\title{
Multi-Ensayos
}

Vol. 7, núm. 13

ISSN: 2412-3285

https://multiensayos.unan.edu.ni

DOI: https://doi.org/10.5377/multiensayos.v7i13.10749

\section{La educación superior y el COVID-19: Experiencias desde mi perspectiva como docente}

\section{COVID-19 and higher education: Experiences from my perspective as a teacher}

\author{
Julio César Roa Rocha ${ }^{1}$
}

Recibido: 17 de agosto de 2020. Aceptado: 28 de septiembre de 2020

\section{RESUMEN}

El siguiente ensayo tiene como propósito crear una reflexión sobre la situación actual desde una perspectiva humanística, educativa y propositiva en relación a la pandemia COVID-19. La actitud reflexiva permite cuestionar las realidades actuales, vencer obstáculos y construir nuevos constructos apoyándose en un pensamiento crítico reflexivo. No es desconocido que el ser humano ha atravesado grandes dificultades a lo largo de su historia, las cuales lo han llevado a generar una sociedad del conocimiento para enfrentar los constantes cambios de un mundo moderno. Para el propósito de este ensayo, la tesis se enfoca en tres puntos vitales: la historia como referente de la evolución humana, los cambios eminentes en la educación superior y consejos para el futuro educacional desde mi perspectiva docente.

Palabras clave: Educación superior; crisis; tecnología; cambios; pandemia.

\begin{abstract}
The following essay aims to create a reflection on the current situation from a humanistic, educational and purposeful perspective in relation to the COVID-19 pandemic. The reflective attitude allows questioning current realities, overcoming obstacles and building new constructs based on reflective critical thinking. It is not unknown that the human being has gone through great difficulties throughout his history, which have led him to generate a knowledge society to face the constant changes of a modern world. For the purpose of this essay, the thesis statement is focused on three vital points: history as a benchmark of human evolution, the eminent changes in higher education and some advice from my standpoint as a teacher.
\end{abstract}

Keywords: Higher education; crisis; technology; changes; pandemic.

1 Universidad Nacional Autónoma de Nicaragua - FAREM-Matagalpa. Correo electrónico: juliorn27@yahoo.com (C) 2021 Revista Multi-Ensayos. 


\section{INTRODUCCIÓN}

Ante la inminente llegada del despertar de la consciencia humana y crítica, los seres humanos se ven implicados en una situación que parece ensañarse en un sector vulnerable de la población; acontecimientos que traen consigo cambios sociales, económicos, educativos y culturales, los cuales podrían tener efectos perniciosos en la población más indefensa.

Estamos ante una pandemia que para muchos puede ser ficción o realidad, el fin de un modelo económico, un sueño, temor u oportunidad; el inicio de una guerra contra la especie humana, un desafío, un arma letal, un virus genéticamente modificado; así como un generador de conflictos emocionales y separaciones, entre tantas corrientes de opiniones y pensamientos, propios de un ser racional y consciente de su entorno.

Existen tantas hipótesis generadas por esta pandemia plagada de infodemia, que hoy afecta al cosmos, donde se cuestiona desde el pensamiento crítico del individuo común hasta a los magnos generadores del conocimiento científico; todos en busca de una solución al problema.

Es un hecho que esta situación de emergencia humanitaria tendrá un trasfondo en todas las esferas sociales. Una situación, que, sin duda alguna, atañe a todos por igual, sin diferencia alguna, cuyas lecciones pueden ser seculares con efectos colaterales nunca antes vividos para muchos. Sin embargo, esta situación puede también ser objeto de cambios estructurales, necesarios quizás, para el desarrollo de un nuevo sistema de vida, donde el hombre se apropie de un rol más consciente dentro del universo. Este ensayo parte de las generalidades que, en la actualidad, causan desasosiego al ser humano; pero centra su tesis en la educación superior y la pandemia actual, tomando tres puntos pivótales: la historia como referente de la evolución humana, los cambios eminentes en la educación superior actual y algunos consejos desde mi experiencia docente.

\section{DESARROLLO}

A lo largo de la historia, la especie humana siempre ha enfrentado obstáculos como catástrofes, cambios climáticos, hambre, guerras, terremotos, huracanes entre otros desafíos que han amenazado nuestra existencia y sostenibilidad en el universo. Dentro de los aportes de la teoría darwiniana (1859), El Origen de las Especies, es bastante común dentro de la literatura encontrarse con un ser que lucha por sobrevivir, mantener su existencia, cambiar particularidades de forma gradual y adaptarse a las diferencias que el medio le presenta.

Darwin (1859), científico influyente por su contribución al conocimiento, reconoce que el individuo evoluciona y se transforma a lo largo del tiempo. Indudablemente, el hombre, como especie, ha sido objeto de cambio evolutivo, ha demostrado ser capaz de enfrentar y luchar contra un sin número de adversidades para mantener su supervivencia. Por aludir un ejemplo, el hombre de la prehistoria, vivía en pequeños grupos que subsistían de la caza y de la pesca, pero en el transcurso del tiempo mejoró sus técnicas de sobrevivencia, aprendió a vivir en sociedades y a trabajar en equipo, lo cual le permitió mejorar su vida (Salas, 2012). Es decir, que el ser se da cuenta que trabajar de forma individual, minimiza 
su supervivencia. A su vez, el uso de otras técnicas no solo le permitió al ser humano alcanzar una mejor vida, sino que lo facultó con oportunidades de transmitir y compartir el nuevo conocimiento dentro de la sociedad. Estamos ante un aprendizaje prácticamente proveniente de la observación y la práctica. Todas estas etapas, sin duda alguna, dan pautas al desarrollo y evolución de la especie humana en lo que respecta al conocimiento y supervivencia.

Para muchos, la historia por sí sola, es una acumulación de sucesos, condicionada por las necesidades y contradicciones sociales como parte de la evolución del sistema. No obstante, la historia debe ser concebida desde una perspectiva crítica donde el hombre analiza la relación y las causas que producen las contradicciones y disconformidades sociales, creadas usualmente por aquellos que gozan del poder y esterilizan el sueño del oprimido (Marx, 1975). Por mencionar un caso, en el antiguo Egipto se cree que la educación era vista en tres niveles, la primera dirigida a los sacerdotes que se ocupaban de enseñar astronomía, la segunda enfocada en oficios menores y la tercera dirigida a la clase dominante centrada en la formación política. Otro dato importante de la educación de la élite egipcia, era la separación entre la oratoria y la escritura. La oratoria estaba orientada a los políticos, quienes necesitaban poseer habilidades extraordinarias del arte de hablar, debido a su constante convivencia con las personas. La escritura, dada su complejidad, requería de habilidades intelectuales que permitieran interpretar imágenes y escribir jeroglíficos. El ejemplo anteriormente compartido tiene como fin enseñarnos cómo la educación fue un eje importante para los que gozaban de ciertos privilegios. Cabe señalar que todo lo referente a la educación egipcia está relacionada a la enseñanza de las élites (Salas, 2012).

Para sustentar y culminar este primer punto de la tesis, me permito citar a Platón quien argumenta que "al conocimiento verdadero sólo se llega por medio de la razón y del entendimiento, y no de la sensación; las cosas no se aprehenden por medio de la experiencia sensible, sino mediante el ejercicio de la razón" (citado en Chacón y Covarrubias, 2012, p.143). Indiscutiblemente, la historia ha sido un referente que nos ayuda a entender los procesos del pasado, recordándonos que somos producto del mismo, pero si somos conscientes, podemos aprender a construir nuestro futuro, tomando como referencia el pasado y el ahora.

Como parte de la historia, el conocimiento ha estado ahí, presente, aportando para la construcción de una sociedad más justa e impulsando un futuro con lecciones aprendidas. En este sentido, examinar la historicidad desde el ámbito educativo, nos permite ver que venimos de un aprendizaje que ha estado en constante evolución. Por ejemplo, en la actualidad, nos encontramos con una educación que ha tenido que migrar abruptamente de la formación presencial a la virtual, creando un cese temporal en las actividades educativas. En ese sentido, IESALC - UNESCO (Instituto Internacional de la UNESCO para la Educación Superior en América Latina y el Caribe (IESALC), reporta que, desde diciembre hasta el 24 de marzo del 2020, "138 países han cerrado escuelas y universidades, impactando más del $80 \%$ de la población estudiantil mundial" (citado en Oliva, 2020, p.13).

A través de la historia, las universidades han tenido que cerrar sus puertas por diferentes epidemias, pero estas dificultades no han evitado que ellas alcancen su misión aun con las puertas cerradas. Por mencionar un ejemplo, en 1665, a raíz de peste negra que golpeó Inglaterra, la universidad de Cambridge cerró sus instalaciones, hecho que sucedió nuevamente en este 2020 (IESALC -UNESCO, 2020). En muchos países 
se tomó la decisión de suspender las actividades, dejando de operar presencialmente. Estas decisiones se dieron con el fin de salvaguardar la salud de los estudiantes, docentes y familias, en un contexto donde los conglomerados de personas ponen en riesgo la salud de otros. El hecho de que no exista exactamente una referencia en relación a cómo enfrentar situaciones como la actual, hace que se torne difícil predecir el futuro inmediato de la educación.

Pedró (2020) argumenta que los cambios en la educación superior han sido grandes, haciendo mención de la calidad, equidad, cambio de metodologías y transición hacia la educación a distancia. Esta última, aunque no es quizás la respuesta optima, se ha convertido en la única solución para muchas instituciones de educación superior. Este mismo autor expone tres razones por la cual se deben esperar resultados negativos en la educación a distancia: la primera es asumir que los docentes y estudiantes poseen los recursos de índole tecnológica y conectividad para hacer frente a este nuevo reto. La segunda es creer que todos los estudiantes están listos para la educación superior a distancia, aun cuando este tipo de aprendizaje tiene algunos años de existencia. Cabe señalar que la educación a distancia es más común en niveles de posgrado. La tercera razón tiene que ver con las competencias de los docentes y estudiantes en un nuevo paradigma, el cual puede llevar a frustración en cuanto a la adaptación a una modalidad educativa diferente; por citar un ejemplo, muchos estudiantes se quejan de recibir información excesiva en las diferentes plataformas educativas.

Con el fin de garantizar el servicio educativo, muchas instituciones, dieron inicio a la utilización de redes sociales como WhatsApp para entablar comunicación con los estudiantes y otras plataformas digitales como Google Classroom y Zoom, que se fueron incorporando durante el proceso. Después de esta crisis y la aceleración de la digitalización, es necesario fortalecer la educación superior a través de la incorporación de novedad tecnológica donde las instituciones dialoguen con lo emergente, sin olvidar los lazos afectivos creados por la educación presencial (Dussel, Ferrante y Pulfer, 2020).

En el caso particular de la educación nicaragüense, hay que reconocer que los docentes buscaron formas de manera rápida para seguir compartiendo conocimiento, aunque hay que aceptar que la educación en línea y a distancia requiere de un nivel de capacidad técnica, conectividad e infraestructura que tristemente no está disponible para muchos estudiantes. Hay muchos lugares donde el internet es inaccesible para una gran parte de la población.

Dentro de este mismo contexto, normalmente los estudiantes ponen recargas en su teléfono para la semana con las diferentes compañías como Claro o Movistar, la cual puede durar poco tiempo, dependiendo del uso que se le dé, caso especial de la realidad de muchos universitarios nicaragüenses. Son pocos los estudiantes que gozan de un plan mensual de uso de internet que le permite tener banda ancha móvil. Cabe señalar que, en el ámbito nicaragüense, muchos estudiantes vienen de la zona rural y tienen que salir de sus hogares un día antes para poder llegar a su centro universitario. A esto se le suma los escenarios de conflictos emocionales, económicos, impregnados de desigualdades, los cuales se vuelven más comunes en la actualidad, y eso que aún desconocemos los desafíos pospandémicos.

Se puede decir que las universidades no estaban listas para asumir cambios abruptos y tomar decisiones en cuestión de semanas. Los docentes y estudiantes no estaban listos para la educación a distancia, 
mediada por la tecnología, sin embargo, todos decidimos subirnos al barco por el compromiso social y moral con la población estudiantil, y crear ambientes de aprendizaje improvisado. De igual forma, hay que reconocer que el confinamiento no será sostenible en el tiempo, sobre todo, para países poco desarrollados.

Tristemente, es común ver cómo la brecha digital expone la desigualdad socioeconómica de la educación a distancia; y no es poco probable que los estudiantes desatendidos y en riesgo no vuelvan a los centros de estudios, cuando estos abran sus puertas nuevamente. Hasta este momento, nos hemos dado cuenta de que los cambios en la educación superior son grandes y traen consigo retos para las casas formadoras, los docentes y estudiantes. Muchos de los retos están ligados a la implementación y acceso a la tecnología. En el caso de los docentes universitarios, según Sáenz y Cira (2020), "diseñar recursos didácticos digitales y explorar nuevas plataformas es uno de los principales retos" (p.5). Los docentes que tienen pocas habilidades en el uso de tecnologías para propósitos educativos, pueden tener más dificultades al adecuar los recursos y contenidos. Por lo antes mencionado, es primordial que el docente reciba apoyo externo en lo concerniente al uso de tecnologías en la educación superior.

ANUIES y Subsecretaria de Educación Superior de la SEP, (Asociación Nacional de Universidades e Instituciones de Educación Superior de la República Mexicana A.C. ANUIES), plantearon algunas medidas para mitigar la situación actual: "trabajo colaborativo e intercambio de experiencias en las buenas prácticas de adecuaciones de materiales, regular las transiciones de modalidad, establecer lineamientos para organizar y operar la docencia virtual, capacitar el personal, solicitar adecuaciones a los programas de estudios" (citado en Sáenz y Cira, 2020, p.5).

Los docentes estamos conscientes de que los cierres de las escuelas crean desigualdades educativas, quizás muchos estudiantes ya no regresen a sus centros de estudios, y no solo por la pandemia, sino por problemas financieros personales o por el miedo a ser contagiado. Sin olvidar que, una posible recesión económica crearía mayor dificultad, en especial en grupos desfavorecidos.

Es un hecho que esta pandemia se ha vuelto un acontecimiento que desafía los saberes, recordándonos que somos una especie vulnerable. Es normal que nos aferremos a lo que conocemos, pero debemos reinventar nuestra educación y ver el lado positivo que la pandemia actual puede ofrecer. Por ende, necesitamos encontrar un modelo pedagógico que nos ayude a contrarrestar esta emergencia humanitaria no prevista.

Aunque cada país tiene un contexto diferente, UNESCO sugiere seguir los principios subsiguientes: asegurar el derecho a la educación, involucrar a los docentes y estudiantes en la búsqueda de respuesta a situaciones de emergencias ante una futura crisis, reflexionar sobre las lecciones aprendidas y rediseñar los procesos de aprendizaje (citado en IESALC-UNESCO, 2020).

Dado todos estos cambios en la educación superior y tomando la historia como referente, me permito compartir algunos consejos desde mi perspectiva docente con el fin de rediseñar el proceso de aprendizaje, tomando en cuenta que podemos volver a vivir otra crisis humanitaria. Primero, tomar en cuenta la experiencia vivida por los docentes y estudiantes, antes de tomar decisiones en cuanto a un cambio de 
modalidad. Hay que reconocer que ambos son los principales actores. Segundo, dar seguimiento a las dificultades y necesidades que ambos actores puedan encontrar en la nueva ruta de aprendizaje virtual. Tercero, reorganizar los calendarios académicos, si fuese necesario y diseñar programas híbridos que combinen actividades presenciales y no presenciales. Para el futuro, se recomienda que las carreras de pregrado contengan asignaturas básicas que puedan ser tomadas de forma virtual para crear un hábito y una transición a la modalidad online. Este ejercicio dará a los estudiantes oportunidades de conocer y dominar el uso de tecnologías con propósitos educativos; por ende, los ayudará a estar listos cuando se presente otra crisis como la actual.

Para finalizar este tercer punto de la tesis, cito a Edgar Morín (citado en UNESCO, 1999) y su gran obra "Los Sietes saberes Necesarios para la Educación del Futuro", enfocándome en dos puntos esenciales que son: enfrentar las incertidumbres y enseñar la comprensión.

Para enfrentar las incertidumbres, primero, hay que iniciar siendo conscientes que predecir la historia humana o lo que pueda pasar con el ser humano el día de mañana, es casi imposible; aun con los grandes avances, decir cómo podrá ser la nueva normalidad es casi un enigma. La historia nos muestra que no solamente las buenas ideas traen cambios, sino que las ideas destructivas pueden ser objeto de modificación, que obliguen al ser humano a encontrar soluciones, las que poco a poco se vuelvan parte de lo cotidiano.

Al enseñar con comprensión, aun con los grandes avances y múltiples progresos de las cuales el ser humano ha sido testigo, todavía encontramos a un ser incomprensivo y más alejado del otro. Una condición fundamental para que se dé la comprensión es la comunicación. Cuando comunicamos debemos de tener en cuenta que nuestra visión puede ser objeto de amenaza para los que temen a lo desconocido.

En el caso particular de la educación nicaragüense, los docentes debemos tomar esta situación actual como una aventura que nos arrastrará a caminos nuevos, a desafíos por descubrir, donde tomaremos roles activos y cuestionaremos lo cuestionable ante un nuevo nacimiento. Lo incierto de la actualidad nos llevará a caminos desconocidos donde la acción será una decisión. El conocer los efectos que provocan los cambios a largo plazo es algo casi difícil de prever. Ante los diferentes acontecimientos, es necesario que el individuo se nutra de comprensión hacia los demás, la cual en un futuro cercano debería ser misión de la educación. Finalmente, es esencial preparar a nuestros jóvenes nicaragüenses con una educación lista para enfrentar obstáculos, como único camino hacia el mundo inteligible.

\section{CONCLUSIÓN}

Para el ser humano es difícil avanzar en su recorrido por el mundo, si olvida el lugar privilegiado que ha jugado la historia, ese referente lleno de experiencias que sirve de brújula en la búsqueda incesante de la felicidad y el buen vivir. El ser humano ha vivido en un mundo incierto, plagado de cambios abruptos, los cuales ha tenido que abrazar y enfrentar con valentía. Las transiciones han sido abismales desde lo conocido hasta lo desconocido, se han afrontado con valor los diferentes cataclismos históricos. 
Es interesante reflexionar que vinimos de un ser individualista que vivía en pequeños grupos hasta llegar a un ser humano con sentimientos, emociones, y muchos deseos de compartir con otros seres; todos en busca de la construcción de una sociedad global. Darwin en su teoría nos enseñaba que el más fuerte sería el vencedor, lo que ha motivado al ser humano a una vida de constante lucha contra los que crean desigualdades.

Finalmente, no olvidemos que cada individuo tiene en sus manos hacer la diferencia para preservar la especie humana, para mantener la educación viva. Los docentes debemos luchar cada día contra las incertidumbres que se presentan y hacer siempre lo mejor que sabemos hacer: "enseñar con amor para una mejor comprensión humana". Debemos recordar que nuestra visión del mundo siempre se verá amenazada por la incomprensión de algunos; en consecuencia, las diferentes reflexiones de vida de la especie humana nos conducen a afrontar la incertidumbre y repensar el porvenir de una educación que contribuya a un futuro viable, donde todos tengan las mismas oportunidades. Morín nos enseñó a ver la educación como un proceso que debe estar en constantes transformaciones, donde se cuestione el cómo enseñamos y qué otros recursos podemos utilizar en nuestra labor docente. ¿Estamos preparados o al menos dispuestos a jugar nuestro rol en el mundo de hoy?

\section{REFERENCIAS}

Chacón, A. y Covarrubias, F. (2012). El sustrato platónico de las teorías pedagógicas. Tiempo de Educar, Vol.13, (25), p.139-159. Recuperado de https://www.redalyc.org/pdf/311/31124808006.pdf

Darwin, C. (1859). On the Origin of Species. New York: D. Appleton and Company.

Dusel, I., Ferrante, P., y Pulfer, D. (2020). La educación de pasado mañana. Notas sobre la marcha. Análisis Carolina,(41), p.1-13. doi:10.33960/AC_41.2020. Recuperado de https://www.fundacioncarolina. es/wp-content/uploads/2020/06/AC-41.-2020.pdf

IESALC-UNESCO. (2020). Covid-19 y educaciòn superior: De los efectos inmediatos al dìa despuès. Análisis de impactos, respuestas políticas y recomendaciones. Recuperado de http://www.iesalc.unesco. org/wp-content/uploads/2020/04/COVID-19-060420-ES-2.pdf

Marx, K. (1975). El capital. Crítica de la economía política. Siglo XXI editores. Recuperado de http://ecopol. sociales.uba.ar/wp-content/uploads/sites/202/2013/09/Marx_El-capital_Tomo-1_Vol-1.pdf

Oliva, H. (2020). La educación en tiempos de pandemias: visión desde la gestión de la educación superior. p.1-16. Recuperado de https://www.researchgate.net/publication/340270478_La_Educacion_en_ tiempos_de_pandemias_vision_desde_la_gestion_de_la_educacion_superior

Pedró, F. (2020). Covid-19 y educación superior en América Latina y el Caribe: efectos, impactos y recomendaciones políticas. Análisis Carolina, (26), p.1-15. doi: 10.33960/AC_36.2020. Recuperado de https://www.fundacioncarolina.es/wp-content/uploads/2020/06/AC-36.-2020.pdf

Salas, J. (2012). Historia general de la educaciòn. México: Red Tercer Milenio S.C. ISBN 978-607-733-032-5. Sàenz, M.y Cira, J. (2020). La Educación Superior en los tiempos del Covid-19; impactos inmediatos, acciones, experiencias y recomendaciones. p. 1-11. Recuperado de https://www.researchgate.net/ publication/341447328_La_Educacion_Superior_en_los_tiempos_del_Covid-19_impactos_ inmediatos_acciones_experiencias_y_recomendaciones

UNESCO (1999). Los sietes saberes necesarios para la educación del futuro. Edgar Morin. 7 place de Fontenoy - 75352 París 07 SP - Francia. 